“C 2017 IEEE. Personal use of this material is permitted. Permission from IEEE must be obtained for all other uses, in any current or future media, including reprinting/republishing this material for advertising or promotional purposes, creating new collective works, for resale or redistribution to servers or lists, or reuse of any copyrighted component of this work in other works." 


\title{
Mutual Coupling Reduction Using Meta-Structures for Wideband, Dual-Polarized, High-Density Patch Arrays
}

\author{
Ming-Chun Tang, Senior Member, IEEE, Zhiyuan Chen, Student Member, IEEE, Hao Wang, Student \\ Member, IEEE, Mei Li, Member, IEEE, Bing Luo, Jiadi Wang, Zhongli Shi, and Richard W. Ziolkowski, \\ Fellow, IEEE
}

\begin{abstract}
A mutual coupling reduction strategy that employs meta-structures is introduced for wideband, dual-polarized, high-density, planar, patch antenna arrays. The meta-structures consist of two types of resonators: grounded capacitively loaded loops (GCLLs) and $\pi$-shaped elements. By incorporating the meta-structures into the array configuration, the isolation levels between adjacent radiating elements in both the $\mathrm{E}$ - and $\mathrm{H}$ - plane orientations are improved by as much as $7.15 \mathrm{~dB}$. The surface current distribution behaviors of the array with only the GCLLs and with only the $\pi$-shaped elements are investigated thoroughly to explain the mutual coupling reduction mechanisms. A proof-of-concept array was constructed and tests were performed that validate the reported design principles and simulation results.
\end{abstract}

Index Terms - Dual-polarized array, high-density array, meta-structure, mutual coupling reduction, patch antenna

\section{INTRODUCTION}

$\mathrm{M}$ ultiple-input-multiple-output (MIMO) technology has become a crucial part of the upcoming fifth-generation

Manuscript received on Jan. 17, 2017, revised on Apr. 07, 2017, and accepted on May 21, 2017.

This work was supported in part by the National Natural Science Foundation of China contract number 61471072, in part by the graduate scientific research and innovation foundation of Chongqing, China contract number CYB16047, in part by the Chongqing Postdoctoral Special Funding Project contract number xm2016022, in part by the China Postdoctoral Science Foundation contract number 2016M590860, in part by the Funding of the Young Backbone Teachers in Colleges and Universities of Chongqing contract number 0307001104102, in part by the Opening subject of State Key Laboratory of Millimeter Waves contract number K201732, and in part by the Australian Research Council grant number DP160102219.

M. -C. Tang is with the Key Laboratory of Dependable Service Computing in Cyber Physical Society Ministry of Education, College of Communication Engineering, Chongqing University and also with the State Key Laboratory of Millimeter Waves, Nanjing, 210096, China (E-mail: tangmingchun@cqu.edu.cn);

Z. Chen, H. Wang, and M. Li are with the College of Communication Engineering, Chongqing University, Chongqing, 400044, China (E-mail: 20151213060@cqu.edu.cn, wangh_2015@cqu.edu.cn, and li.mei@cqu.edu.cn);

B. Luo, J. Wang, and Z. Shi are with Huawei Technologies Co. Ltd (E-mail: roben.luo@huawei.com, wangjiadi@huawei.com,

and shizhongli@huawei.com);

R. W. Ziolkowski is with the University of Technology Sydney, Global Big Data Technologies Centre, Ultimo NSW 2007, Australia, and the Department of Electrical and Computer Engineering, University of Arizona, Tucson, AZ 85721, USA (E-mail: Richard.Ziolkowski@uts.edu.au)
(5G) communication systems $[1,2]$. It has several unique advantages that arise from its ability to facilitate the presence of additional signal channels instead of requiring the use of extra frequency spectrum or power. Benefits include being able to increase the data capacity and to make the system more adaptable.

MIMO approaches are based on antenna arrays. With the ever increasing demand for more capacity, it is anticipated that massive MIMO will be central to 5G systems; and it will be facilitated by compact dense arrays. However, strong inter-element coupling occurs when the element spacing is small; it dramatically increases the spatial correlations and seriously deteriorates the signal-to-interference-plus-noise -ratio (SINR) [3]. Consequently, mutual coupling effects between array elements have attracted intense attention.

There exist a plethora of reported approaches that are capable of reducing the mutual coupling in the physical layer. However, because of known fundamental physical limitations [4], it cannot be eliminated completely. Nevertheless, mutual coupling reduction can significantly improve the performance of MIMO systems.

In general, the existing, most widely used mutual coupling reduction strategies can be classified into the following three categories. One approach focuses on introducing a variety of compact isolation elements between the radiators. The choices have been rather diverse. They include parasitic elements, such as monopoles [5], scatterers [6], and radiation patches [7]. Also, they include electromagnetic band-gap (EBG) structures, such as mushroom-like EBG structures [8], F-shaped EBG structures [9], and uniplanar compact EBG structures [10, 11]. Defected ground structures (DGSs), such as periodic rectangular slits [12], back-to-back U-shaped slots [13], T-shaped slots [14], and loop slots [15] have also been used. More recently, numerous metamaterial-inspired structures have been considered, such as folded single split ring resonators [16], grounded split-ring resonators (GSRRs) [17], multiple split-ring resonators (MSRRs) [18], complementary split-ring resonators (CSRRs) [19], elliptical split-ring resonators (E-SRRs) [20], embedded circuit (EC) resonators [21], capacitively loaded loop (CLL) resonators [22], waveguide-based resonators [23], composite metamaterials [24-25], artificial magnetic conductor (AMC) meta-structures 
[26, 27], double-layer mushroom structures[28], and coplanar strip walls [29]. Several types of transmission lines that directly connect adjacent antenna elements have also been applied to reduce the mutual coupling. These transmission lines provide effective decoupling current pathways; examples include neutralization lines [30-32] and feedforward lines [33]. A second class of mutual coupling suppressors relies on the construction of decoupling networks behind the radiators. There are two versions of these networks, i.e., distributed [34-37] and lumped [38] ones. A third set of mutual coupling reducers involves simply rearranging the radiator positions within the array without resorting to any auxiliary structure. Examples include locating the radiating elements near the ground edges [39] and placing them with a certain angular offset [40].

While the above strategies have reduced mutual coupling, one witnesses certain drawbacks accompanying each of them. As a result, their widespread use in engineering applications has not occurred. For instance, most of the isolation elements are effective in only one specific direction. This specificity limits their applications to only single-polarized arrays; it is ineffective for dual-/multi-polarized arrays [5-33]. The decoupling networks suffer from quite narrow bandwidths and, hence, cannot be applied to wideband arrays [34-38]. The ground plane related strategies can only be utilized when the ground planes are limited in size and, hence, they cannot be applied to large arrays [39].

Consequently, if it were possible, it would be highly desirable to achieve a mutual coupling reduction approach that not only would be suitable for dual-/multi- directions, but also for large arrays in a wideband frequency range. It should be noted that a two-element, dual-polarized, magnetically coupled, wideband patch antenna with high element isolation has been accomplished [41]. However, the distance between adjacent elements is quite large $\sim 1.3 \lambda_{0}$, where $\lambda_{0}$ indicates the operational wavelength in the free space. While this size factor leads to an inescapable appearance of grating lobes in potential phased array applications, it also negates the use of this approach for compact arrays.

We report in this article a mutual coupling reduction method based on meta-structures that significantly enhances the performance characteristics of wideband, dual-polarized, high-density, planar patch antenna arrays. The behaviors of two types of resonant meta-structures are discussed first in Section II. These are the grounded capacitively loaded loops (GCLLs) and the $\pi$-shaped elements. In Section III, it is demonstrated that the proposed decoupling meta-structures are effective to reduce the mutual coupling between arrays of any lattice configuration or polarization. The performance of two-element arrays with only the resonant GCLLs and with only the resonant $\pi$-shaped elements are investigated separately to provide insights into their mutual coupling reduction mechanisms. A prototype system was fabricated and measured. The experimental setup and the measured results are described in Section IV. The experiments validated the design principles, and the measured results agree well with the corresponding simulated values. Finally, some conclusions are drawn in section $\mathrm{V}$.
We note that all of the metallic elements modeled in the antenna designs were chosen to be copper with its known material parameters: $\varepsilon_{r}=1.0, \mu_{r}=0.999991$ and bulk conductivity $\sigma=5.8 \times 10^{7} \mathrm{Siemens} / \mathrm{m}$. All of the numerical simulations and their optimizations were performed using the frequency domain, finite element-based ANSYS/ANSOFT High Frequency Structure Simulator (HFSS), version 15.0 [42]. A $50 \Omega$ source impedance is assumed throughout.

\section{Performance Characteristics of the META-STRUCTURES}

Two types of resonant meta-structures are introduced for the mutual coupling reduction. These are the GCLLs and the $\pi$-shaped elements. After initial consideration of their individual impact on the high-density array characteristics, their mutual integration into the array and the resulting performance enhancements will be described.

\section{A. GCLL resonator}

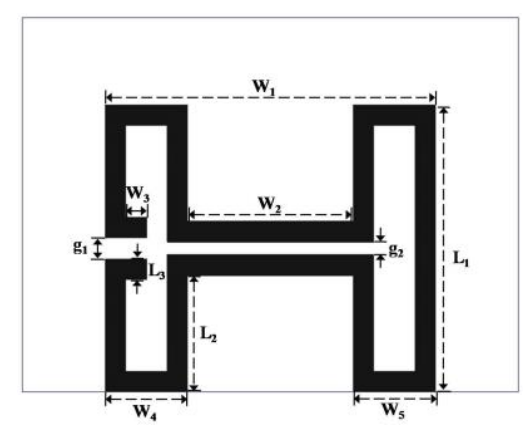

(a)

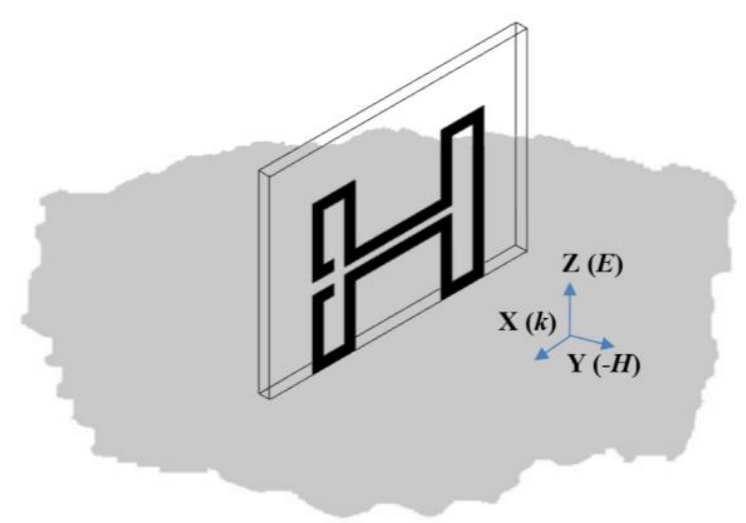

(b)

Fig. 1 The GCLL meta-structure configuration. (a) The physical geometry with its defining parameters, and (b) the unit cell simulation model.

TABLE I. THE DESIGN PARAMETERS OF THE GCLL ELEMENT (All Dimensions ARE in Millimeters)

\begin{tabular}{|c|c|c|c|c|}
\hline$L_{1}=6.9$ & $L_{2}=2.8$ & $L_{3}=0.5$ & $W_{l}=8$ & $W_{2}=4$ \\
\hline$W_{3}=0.5$ & $W_{4}=2$ & $W_{5}=2$ & \multicolumn{2}{|c|}{ Null } \\
\hline
\end{tabular}

The configuration of one GCLL unit cell, together with its geometric parameters, is provided in Fig. 1(a). The CLL structure is oriented orthogonal to and is connected directly to the ground plane. The metallic traces of the CLL structure are 
supported on an Arlon $\mathrm{AD} 450^{\mathrm{TM}}$ substrate with relative permittivity: $\varepsilon_{r}=4.5$, and loss tangent: $\tan \delta=0.0035$. The thickness of the substrate is $0.508 \mathrm{~mm}$. Table I lists its optimized parameter values. Fig. 1(b) represents the simulation model; it indicates the EM environment imposed on the GCLL element, i.e., it is illuminated by a plane wave propagating along the $x$-axis with its electric ( $E$-) field being parallel to the $z$-axis and its magnetic $(H-)$ field being parallel to the $y$-axis.

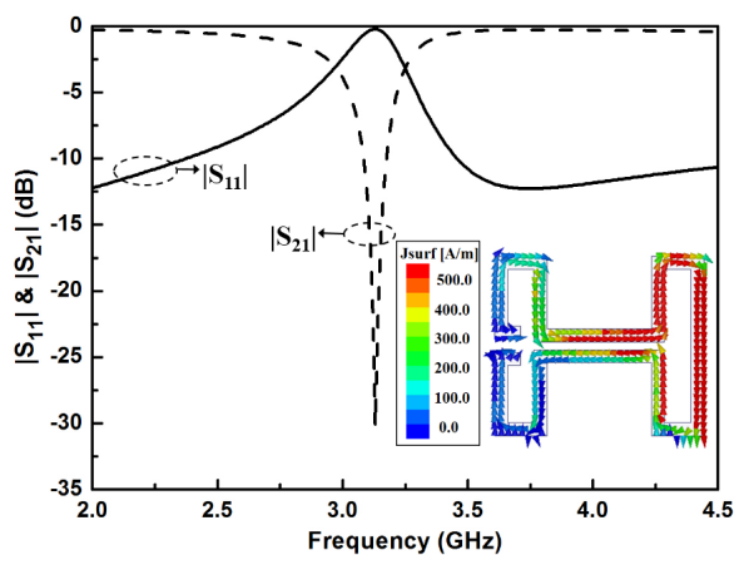

(a)

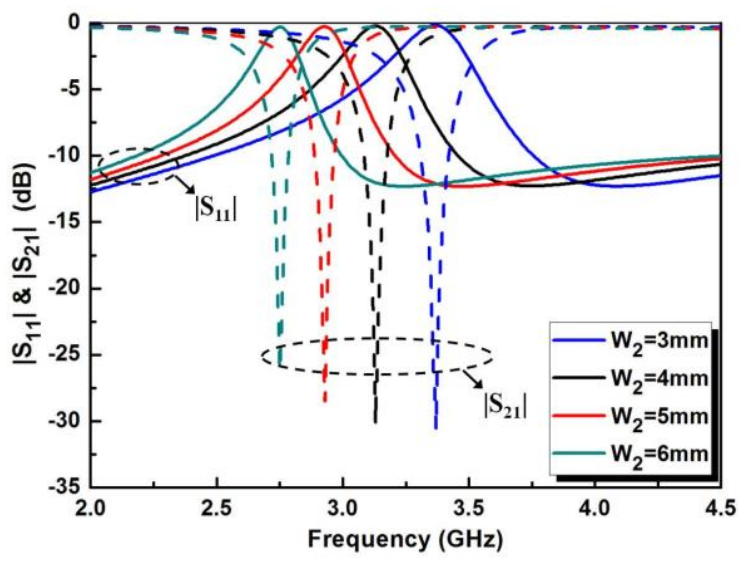

(b)

Fig. 2 Simulation results of the GCLL meta-structure. (a) S-parameters of the configuration in Fig. 1, and (b) the results of the parametric study of the meander line length, $\mathrm{W}_{2}$.

Details of the HFSS simulations are as follows. Perfect electric conducting (PEC) and perfect magnetic conducting (PMC) boundary conditions were imposed in the $z$ and $y$ directions, respectively. Two excitation ports were assigned in the $x$ direction. The simulated reflection and transmission properties of this GCLL meta-structure are shown in Fig. 2(a). Excellent isolation performance is exhibited and the peak isolation value is above $30 \mathrm{~dB}$ at the resonance frequency, $3.135 \mathrm{GHz}$. The surface current density behavior at the resonance frequency is similar to the folded single split ring resonators and GSRRs reported, respectively, in [16] and [17]. They indicate that the GCLL has a strong electric response behavior [43, 44], which is illustrated in detail in the Appendix. It is noted that there is a pair of meander lines on the upper and lower arms of the GCLL; this structure is thus different from the folded single split ring resonator [16] and GSRRs [17]. The presence of the meander lines not only makes the GCLL unit cell electrically smaller (10.29\% reduction), but they also provide more freedom to adjust its resonance frequency range. As shown in Fig. 2(b), the resonance frequency shifts from 2.75 to $3.37 \mathrm{GHz}$ as the meander line length changes from 3 to 6 $\mathrm{mm}$.

\section{B. $\pi$-shaped resonator}

As is illustrated in Fig. 3(a), a $\pi$-shaped resonator is printed on an Arlon AD450 ${ }^{\mathrm{TM}}$ substrate with the same thickness as that of the GCLL resonator in Fig. 1. Its optimized geometry parameter values are listed in Table II. The corresponding simulation model and the imposed EM environment are shown in Fig. 3(b). Three $\pi$-shaped unit cells are illuminated by a plane wave propagating along the $x$-axis with its $E$-field parallel to the $y$-axis and its $H$-field parallel to the $z$-axis. The HFSS simulation setup has PEC and PMC boundary conditions imposed again in the $y$ and $z$ directions, respectively. Two excitation ports are assigned again in the $x$ direction.

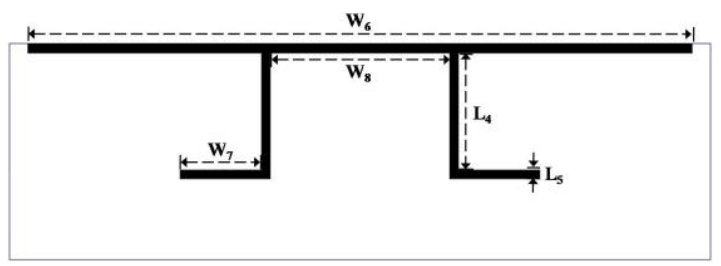

(a)

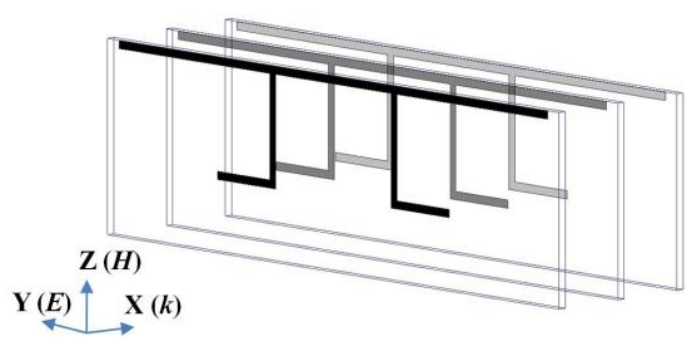

(b)

Fig. 3 The $\pi$-shaped meta-structure configuration. (a) The physical geometry of the unit cell with its defining parameters, and (b) the multiple unit cell simulation model.

TABLE II. THE DESIGN PARAMETERS OF THE П-SHAPED RESONATOR (ALL DIMENSIONS ARE IN MILLIMETERS)

\begin{tabular}{|l|l|l|l|l|}
\hline$L_{4}=6.5$ & $L_{5}=0.5$ & $W_{6}=39$ & $W_{7}=4.5$ & $W_{8=} 10$ \\
\hline
\end{tabular}

The simulated reflection and transmission properties of the resulting bulk metamaterial structure indicate that there is a strong bandgap behavior around 3.14 GHz. As indicated in Fig. 4(a), the peak isolation level at the resonance frequency is $\sim 45$ $\mathrm{dB}$. Note that there is another resonance peak near a lower frequency, $2.83 \mathrm{GHz}$, which is due to the capacitive coupling effect arising from the presence of multiple $\pi$-shaped resonators [45]. The current distribution inset in Fig. 4(a) also indicates that the surface currents on each resonator at the resonance frequency are in phase with the $E$-field of the exciting plane 
wave and, hence, also exhibit an electric response. The effective permittivity and permeability of this bulk metamaterial are also discussed in the Appendix. It should be emphasized that this specially engineered $\pi$-shaped resonator is different from the common wire resonator [43]. Benefiting from its two-leg configuration, one can shift its resonance frequency by only changing the distance $\left(\mathrm{W}_{8}\right)$ between the two legs. The results of the parametric study of $\mathrm{W}_{8}$ are summarized in Fig. 4(b). They indicate that by varying the distance $\mathrm{W}_{8}$ one can shift the resonance frequency in a large frequency interval: 2.6-3.2 GHz.

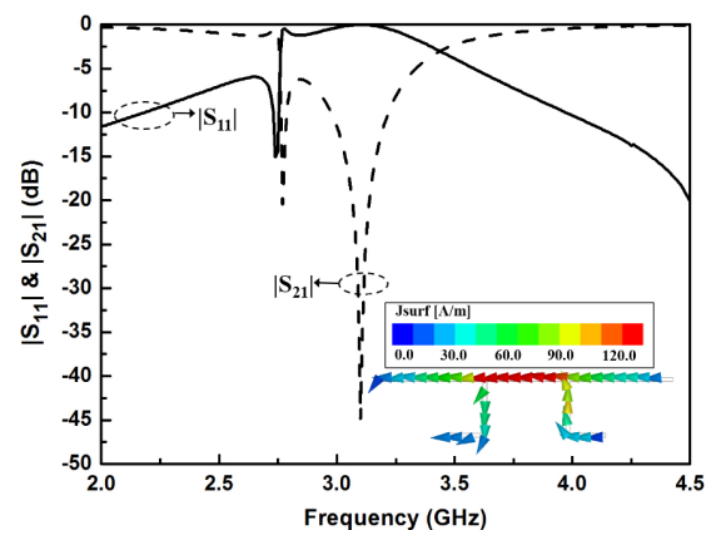

(a)

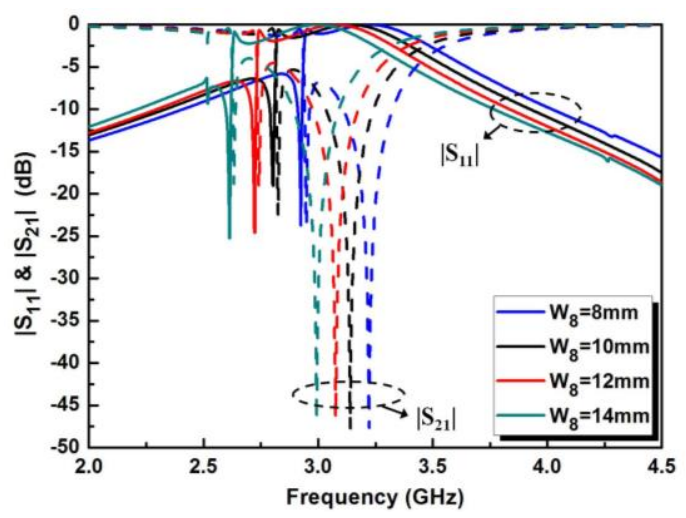

(b)

Fig. 4 Simulation results of the $\pi$-shaped resonator. (a) S-parameters of the bulk metamaterial configuration in Fig. 3(b), and (b) the results of the parametric study of the distance, $\mathrm{W}_{8}$, between the two legs of each unit cell.

It is emphasized that the method of placing a metamaterial unit cell in a simple PEC-PMC parallel plate waveguide (PPW) models the excitation of an infinite array of these elements with a normally incident plane wave. It is a very common technique used to determine the electric and magnetic resonances and responses of a meta-structure, including its reflection and transmission performance characteristics [43, 44]. However, the electromagnetic environment associated with an actual antenna array is much more complex. In order to measure the actual bandwidths of the finite meta-structure more accurately, the two-element probe-fed patch array (with the inter-element distance $\sim 0.46 \lambda_{0}$ ) is studied numerically with and without the meta-structures being present. According to our simulation results, the relative fractional bandwidth of the optimized meta-structures is $\sim 18 \%$ and the mutual coupling reduction is larger than $5 \mathrm{~dB}$ over the entire bandwidth.

\section{MEtA-STRUCTURE MUtUAL COUPLING SUPPRESSION IN AN ARBITRARY LATTICE PATCH ARRAY}

In this section, the proposed meta-structures will be employed in a dual-polarized two-element array to validate their mutual coupling suppression effects.

A. Planar, dual-polarized, slot-coupled, patch antenna element design

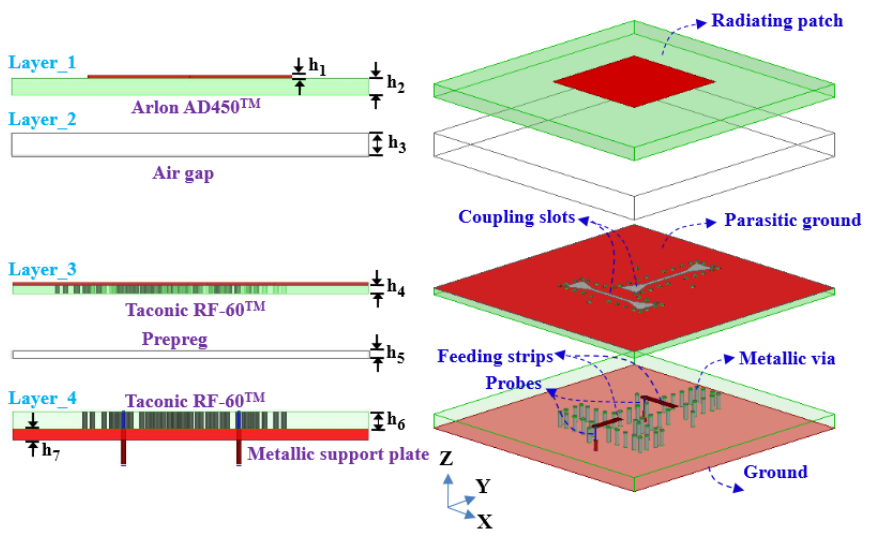

(a)

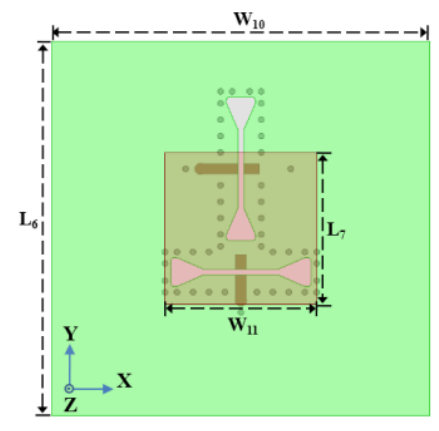

(c) (b)

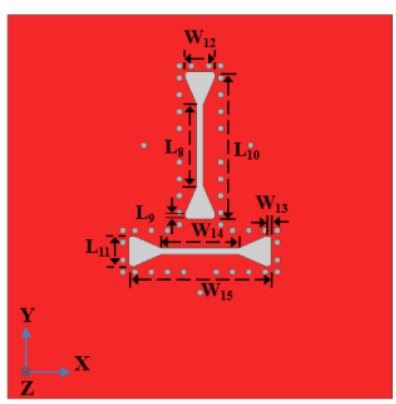

(d)

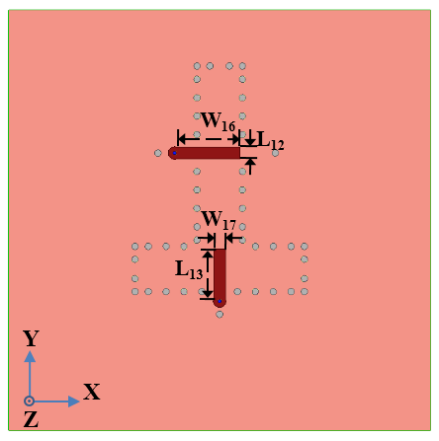

(e)

Fig. 5 Geometry of the dual-polarized, aperture-coupled, patch antenna element. (a) Side view; (b) 3-D view of the antenna with its layers detached; and top views of (c) layer_1, (d) layer_3, and (e) layer_4.

The dual-polarized patch antenna element is shown in Fig.5. It employs an aperture-coupled feeding technology. The 
optimized geometrical parameter values are listed in Table III. The proposed antenna consists of four layers. On the layer_1, a rectangular radiating patch whose size is $\mathrm{L} \times \mathrm{W}$ lies on the top side of an Arlon AD450 substrate with a thickness $\mathrm{h}_{2}=1 \mathrm{~mm}$. An air-gap is placed underneath the patch to serve as the layer_2; it helps control the capacitive coupling between the patch and the pair of coupled slots that are etched orthogonally on the layer_3, the upper face of a Taconic RF-60 substrate with relative permittivity of $\varepsilon_{r}=6.15$ and loss tangent $\tan \delta=$ 0.0028 . Note that, the locations of the two coupled slots relative to the radiating patch are not identical. One slot is totally covered by the patch, while the other is not. This arrangement is advantageous for impedance matching in a wide frequency range, which has been widely applied [46, 47]. Finally, at the layer_4, two metallic strips with orthogonal placement are printed on the top side of a Taconic RF-60 substrate; their perpendicular orientation accommodates the excitation of the

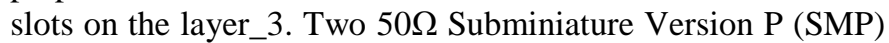
connectors are vertically connected to the two strips from the source.

Notice that there are several metallic vias in the design. They each have a $0.5 \mathrm{~mm}$ radius and connect the top side of layer_3 and the bottom side of layer_4. These connections create a conducting cavity that can be viewed as being formed with substrate integrated waveguide (SIW) technology. This SIW cavity isolates the feed components and concentrates the electromagnetic fields within it. As a result, the presence of the vias not only connects the parasitic ground and the bottom ground plate to improve the antenna gain, but it also enhances the isolation between the two ports. Therefore, this dual-polarization feed structure produces high isolation and radiation levels for the two orthogonal linear-polarization (LP) operations. In addition, an aluminum plate which is the same size as all of the other plates is introduced to act as the ground plate and provides mechanical support for the whole antenna system. It has enough thickness, $\mathrm{h}_{7}=5 \mathrm{~mm}$, to ensure that mechanical stability.

TABle III. Design PARAMETERS OF the PATCh ANTENNA ElEMENT (All Dimensions ARE IN Millimeters)

\begin{tabular}{|c|c|c|c|c|}
\hline$h_{1}=0.017$ & $h_{2}=1$ & $h_{3}=5.35$ & $h_{4}=0.64$ & $h_{5=} 0.053$ \\
\hline$h_{6}=3.18$ & $h_{7}=5$ & $h_{8}=15.5$ & $L_{6}=65$ & $L_{7}=26.3$ \\
\hline$L_{8}=13.8$ & $L_{9}=1.1$ & $L_{10}=24.9$ & $L_{11}=5$ & $L_{12}=1.75$ \\
\hline$L_{13}=7.6$ & $W_{10}=65$ & $W_{11}=26.3$ & $W_{12}=5$ & $W_{13}=1.05$ \\
\hline$W_{14}=13$ & $W_{15}=24.1$ & $W_{16}=9.64$ & $W_{17}=1.75$ & Null \\
\hline
\end{tabular}

In preparation for the explanations of how the mutual coupling reduction is realized, the known $E$-field distributions in the near field of a traditional microstrip patch antenna were taken into consideration [4, 48]. They are illustrated in Fig.6. Three zones (zones (1), (2) and (3), are emphasized. They are divided according to the characteristics of the $E$-field distributions in them. The areas at the two edges of the patch in the $E$-plane (along the $x$-axis) are labeled as zone (1). The areas at the two edges of the patch in the $H$-plane (along the $y$-axis) are labeled as zones (2) and (3), where (2) and (3) indicate, respectively, the areas lower and higher than the patch height.
The corresponding $E$-field behavior in zone (1) is represented by the blue curves; it is represented by the green curves in zone (2); and it is represented by the red curves in zone (3).

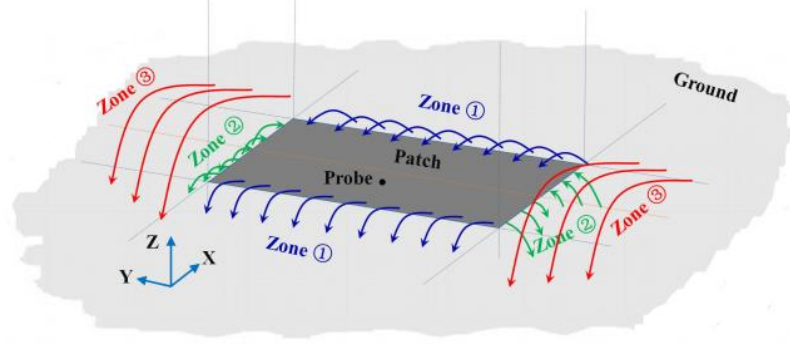

Fig. $6 E$-field distributions in the near field of a microstrip patch antenna.

\section{B. Mutual coupling reduction effects}

The diagram representing a dual-polarized array with an arbitrary two-dimensional lattice arrangement is shown in Fig. 7(a). The cross shapes, which are composed of the red and black short line segments, represent its dual-polarized antenna elements that are linearly polarized in the $x$ and $y$ directions, respectively. Parameters $d_{x}, d_{y}$, and $d$ are, respectively, the inter-element spacing values along the $x$ and $y$ axes and the offset distance along the $y$-axis. When $d$ decreases from a certain value to zero, the array then becomes a common rectangular array, as shown in Fig. 7(b). Therefore, by varying the offset distance $d$, arrays with arbitrary lattice arrangements can be formed.

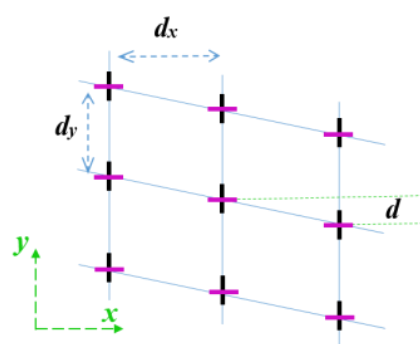

(a)

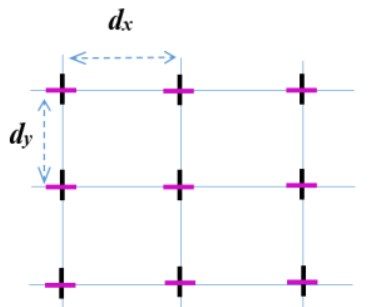

(b)
Fig. 7 Diagram of dual-polarized arrays with (a) arbitrary and (b) rectangular lattice arrangements.

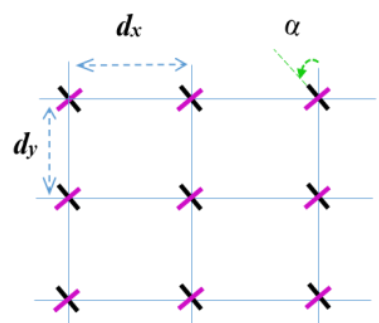

Fig. 8 Diagram of dual-polarized arrays with arbitrary element polarization orientation.

Fig. 8 shows the rectangular array configuration composed of dual-polarized elements rotated by the angle $\alpha$ compared 
with those shown in Fig. 7(b). By varying the rotation angle $\alpha$, a dual-polarized array with a specified polarization orientation can be achieved. In order to investigate the mutual coupling reduction effects of the identified meta-structures in a dual-polarized array defined by an arbitrary lattice array with an arbitrary orientation of its elements, a set of meta-structure loaded two-element arrays having different offset distances $d$ and rotation angles $\alpha$ was analyzed.

\section{(1) Mutual coupling reduction effects with varying offset d}

Fig. 9 depicts a two-element array consisting of two dual-polarized elements, No. 1 and No. 2, with four ports numbered " 1 ", " 2 ", " 3 " and " 4 ". The decoupling meta-structures, i.e., seven resonant GCLLs and three resonant $\pi$-shaped elements, are loaded halfway between the two radiating elements. As shown in Fig. 9(c), the GCLLs are incorporated into the layer_2 and are connected to the parasitic ground on the layer_3. The three $\pi$-shaped elements are placed vertically on the top side of the GCLLs. Note that because of the mutual interaction between the antenna elements and these meta-structures, the resonance frequencies of both types of resonators are blue-shifted slightly away from $3.1 \mathrm{GHz}$. In order to recover slightly lower individual resonance frequencies and to ensure the necessary overlap with the patch antennas' operational frequency range, the two key design parameters: $\mathrm{W}_{2}$ and $\mathrm{W}_{8}$, of each meta-structure were retuned, respectively, to be $11.5 \mathrm{~mm}$ and $16.0 \mathrm{~mm}$.

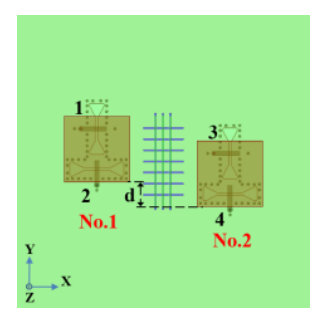

(a)

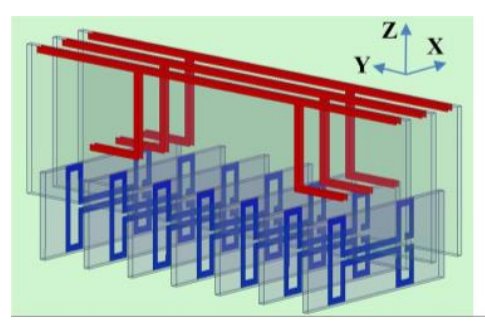

(b)

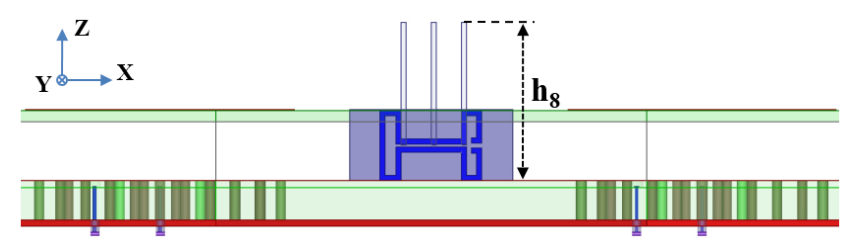

(c)
Fig. 9 Two-element array loaded with decoupling meta-structures having an offset distance $d$. (a) Top view of the array, (b) 3-D zoom-in view of the meta-structures, and (c) side view of the array with the decoupling meta-structures present.

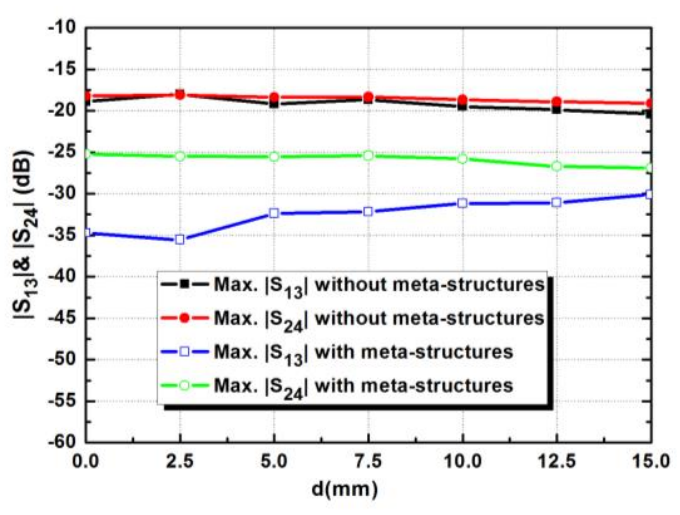

Fig. 10 Simulated S-parameters as functions of the offset distance $d$ for the array with and without the decoupling meta-structures.

Referring to Fig. 9(a), recall that an antenna array with an arbitrary lattice arrangement can be formed by varying its lattice spacings and by varying the vertical distance $d$ between its two elements along the y-axis. Fig. 10 plots the port isolation levels of the co-pol fields $\left(\left|S_{13}\right|\right.$ and $\left.\left|S_{24}\right|\right)$ between the two radiating elements as functions of $d$ for the cases with and without the decoupling meta-structures. Only the mutual coupling suppression effects between the co-polarization (co-pol) ports are examined here, since the port isolation levels of the cross-polarization (cross-pol) fields $\left(\left|S_{14}\right|\right.$ and $\left.\left|S_{23}\right|\right)$ are far below those of the co-pol. All of the isolation levels given in Fig. 10 are the maximum value for each $d$ across the entire frequency band. It is immediately apparent that without the decoupling meta-structures, the isolation levels between the two co-polarized ports decline only very slightly with an increase of $d$ and generally remain above $-20 \mathrm{~dB}$ [24]. In contrast, after loading the array with the decoupling meta-structures, the port isolation of the co-pol fields has been significantly improved for all values of $d$. In particular, $\left|\mathrm{S}_{13}\right|$ $\left(\left|\mathrm{S}_{24}\right|\right)$ shows a minimum reduction of $9.71 \mathrm{~dB}(7.01 \mathrm{~dB})$, yielding all coupling levels below $-25 \mathrm{~dB}$.

(2)Mutual coupling reduction effects with varying rotation angle $\alpha$

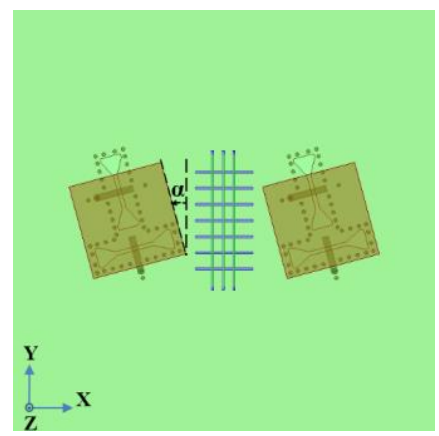

Fig. 11 Two-element patch array loaded with decoupling meta-structures that are oriented with respect to the patches by the rotation angle $\alpha$.

The two-element array with different LP element orientations was analyzed to examine the mutual coupling suppression effects of the proposed meta-structures. As shown 
in Fig. 11, dual-polarized antennas are again employed as the elements of the array. The meta-structures' positions and orientations remained the same. The radiating patch elements were rotated by the angle $\alpha$ relative to the meta-structures.

Fig. 12 plots the port isolation levels between the co-pol ports of the two-element array as functions of the rotation angle $\alpha$ for the cases with and without the decoupling meta-structures. One finds the isolation curves, $\left|S_{13}\right|$ and $\left|S_{24}\right|$, are symmetrical with respect to the $\alpha=45^{\circ}$ case. This occurs because of the orthogonal polarization property of the two co-pol ports. It is obvious that the port isolation level between them remains above $-20 \mathrm{~dB}$ as $\alpha$ increases if the decoupling meta-structures are not present. In contrast, when they are present, the co-pol port isolation levels are dramatically improved across the entire range of $\alpha$ values. In particular, both $\left|S_{13}\right|$ and $\left|S_{24}\right|$ show a minimum reduction of $6.48 \mathrm{~dB}$ and all the coupling levels are again below $-25 \mathrm{~dB}$. Therefore, it has been clearly demonstrated that these decoupling meta-structures are an effective means to reduce the mutual coupling between the elements of a dual-pol patch array in any lattice configuration or with any orientation of its LP elements.

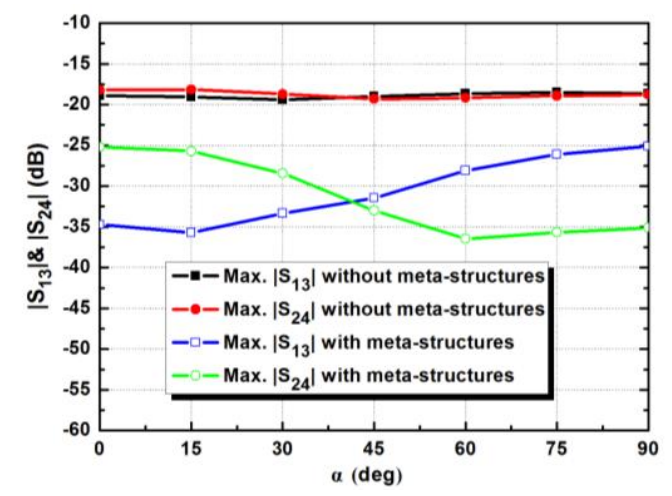

Fig. 12 Simulated S-parameters as functions of the rotation angle $\alpha$ for the dual-polarized array with and without the decoupling meta-structures.

\section{Mutual coupling reduction mechanisms}

In order to further understand the mutual coupling reduction mechanisms, a two-element array with the meta-structures present and with $\mathrm{d}=7.5 \mathrm{~mm}$ and $\alpha=0^{\circ}$ is analyzed in detail as a proof-of-principle example. This lattice configuration corresponds to a closely-spaced triangular array with inter-element spacings of $43.42 \mathrm{~mm}$ and $32.81 \mathrm{~mm}$ along its $\mathrm{x}$ and $y$ - axes, respectively. The simulated $\mathrm{S}$-parameter results of the optimized array with and without the decoupling meta-structures are given in Figs. 13 and 14, respectively. The simulated reflection coefficients of all the ports, as well as the port isolation levels, are shown in Figs. 13(a) and 14(a), respectively. It is obvious that both patch elements exhibit good impedance matching in the range from 3.3-3.6 GHz, where the reflection coefficient $<-10 \mathrm{~dB}$. They also exhibit excellent isolation levels for the cross-pol ports, i.e., they are as high as $35 \mathrm{~dB}$ for both elements No. $1\left(\left|\mathrm{~S}_{12}\right|\right)$ and No. $2\left(\left|\mathrm{~S}_{34}\right|\right)$. This outcome demonstrates that the presence of the decoupling meta-structures has little effect on the S-parameters of each antenna element. The port isolation levels between elements No. 1 and No. 2 are revealed by their co-pol $\left(\left|S_{13}\right|\right.$ and $\left.\left|S_{24}\right|\right)$ and cross-pol $\left(\left|S_{14}\right|\right.$ and $\left.\left|S_{23}\right|\right)$ port levels for the cases with and without the decoupling meta-structures and are shown in Figs. 13(b) and 14(b), respectively, as functions of the source frequency.

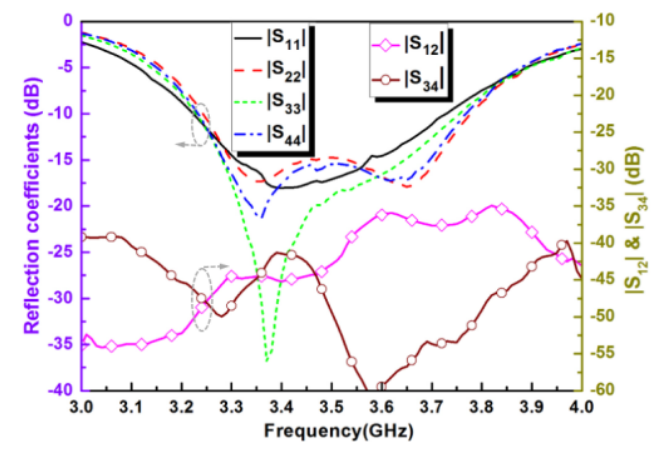

(a)

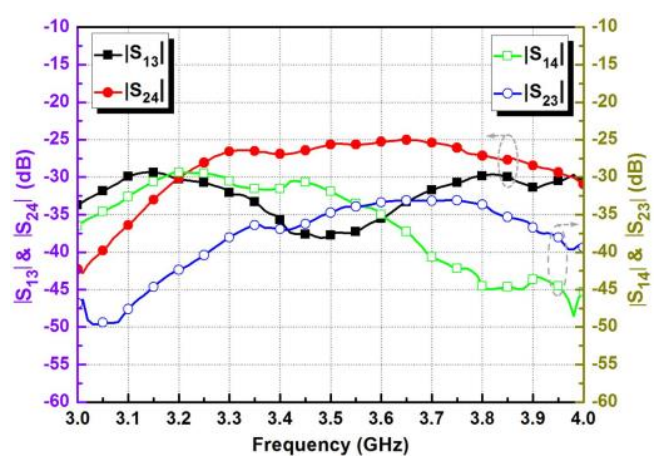

(b)

Fig. 13 Simulated S-parameters for the array with the decoupling meta-structures present as functions of the excitation frequency. (a) Reflection coefficients and port isolation levels for each antenna element, and (b) port isolation levels between the two antenna elements.

It is readily observed that the port isolation of the same polarization has been significantly improved over the entire operational band. In particular, $\left|\mathrm{S}_{13}\right|\left(\left|\mathrm{S}_{24}\right|\right)$ is decreased from $-18.67 \mathrm{~dB}(-18.36 \mathrm{~dB})$ to $-32.19 \mathrm{~dB}(-25.42 \mathrm{~dB})$, witnessing a reduction in the coupling levels of $\sim 13.52 \mathrm{~dB}(\sim 7.06 \mathrm{~dB})$. Taking the differences between the values in Figs. 13 and 14, it is determined that the relative fractional bandwidth, where the isolation between both polarizations has been enhanced by up to $5 \mathrm{~dB}$, is about $23.37 \%$. Moreover, the cross-pol port isolation levels between elements No. 1 and No. 2 ( $\left|S_{23}\right|$ and $\left.\left|S_{14}\right|\right)$ remain very low and almost unchanged. In comparison with most previously reported isolation elements and strategies, which are effective in only one specific direction [5-33], use high-Q decoupling networks [34-38], or introduce modified ground planes [39], our decoupling approach is suitable over a wide frequency range for both polarizations and for large arrays with arbitrary lattice configurations. Furthermore, when compared to a recently reported dual-polarized array decoupling technology that depends on a $\sim 1.3 \lambda_{0}$ inter-element separation [41], our approach with its much smaller 
inter-element distance, $\sim 0.6 \lambda_{0}$, is more suitable for high-density arrays.

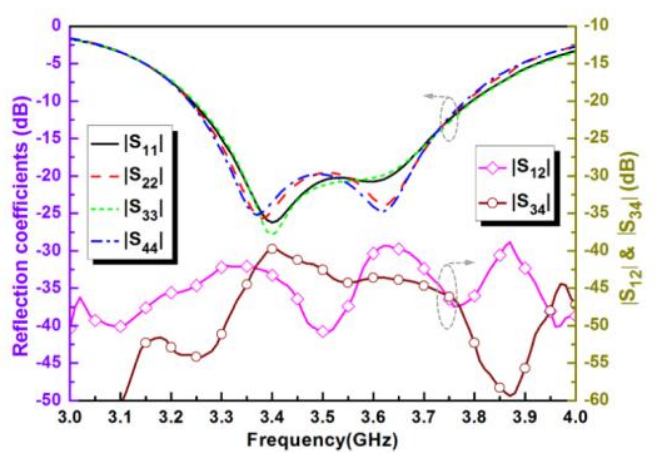

(a)

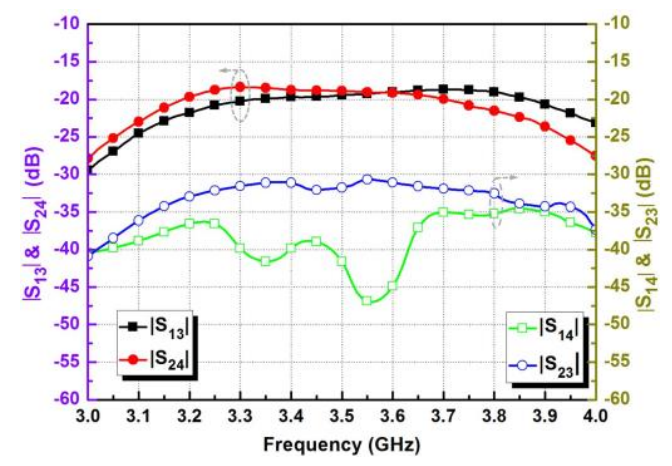

(b)

Fig. 14 Simulated S-parameters for the array without the decoupling meta-structures present as functions of the excitation frequency. (a) Reflection coefficients and port isolation for each antenna element, and (b) port isolation levels between the two antenna elements.

Since the meta-structures illustrated in Fig. 9 consist of two types of metamaterial resonators, i.e., the GCLLs and the $\pi$-shaped elements, the operational mechanisms that lead to the suppression of the mutual coupling associated with each type are described further. To understand these mechanisms, the surface current distributions on the patch elements and the two types of meta-structures are examined separately by loading the radiating elements first only with the GCLLs and then only with the $\pi$-shaped elements.

\section{(1)Two-element array loaded only with GCLLs}

Fig. 15 shows the current distributions when the array is loaded with only GCLLs. As is illustrated in Fig. 15(a), when ports 1 and 3 are excited with the same phase, which is equivalent to the two patches being oriented in their $E$-planes [49], very strong induced currents appear on the GCLL surfaces. The currents densities are also in phase, indicating that the strong electric response $[43,44]$ noted for the GCLL unit cell in Fig. 1 is the main physical mechanism controlling the mutual coupling. In particular, referring to zone (1) in Fig. 6, the strong coupling to the meta-structures shown in Fig. 15(a) originates from the $E$-field behavior between the patches. The electric fields radiated by the patch antennas contain both $E_{x}$ and $E_{z}$ components in their near fields. The presence of the $E_{z}$ component is consistent with the electromagnetic environment associated with the original analysis of the GCLL unit cell; and thus, because they are resonant elements, the same electric bandgap characteristics are manifested. As shown in Fig. 16, the presence of the GCLL meta-structures has decreased the mutual coupling level associated with this $E$-field coupling, i.e., $\left|\mathrm{S}_{13}\right|$, by $5.72 \mathrm{~dB}$ to a maximum level of $-24.39 \mathrm{~dB}$ across the entire operational band. Thus, the presence of the meta-structures produces a significant reduction of the mutual coupling when compared to the results shown in Fig. 14(b) for the array without the GCLL elements. Additionally, it is noted that the GCLL's meander-line features also provide an extra degree of freedom to improve the $\left|S_{13}\right|$ (decoupling) levels even further.

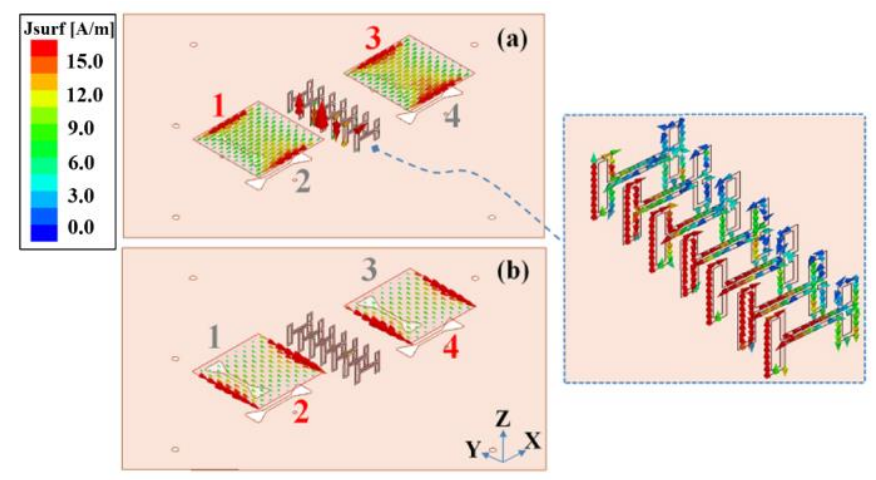

Fig. 15 Surface current distributions on the traces of the two-element array consisting of only elements No. 1 and No. 2 and loaded only with GCLLs. Excitation of (a) ports 1 and 3 only, and (b) ports 2 and 4 only.

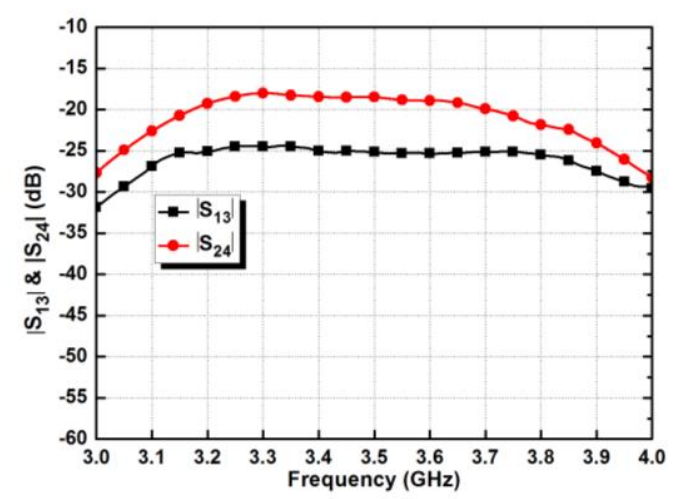

Fig. 16 Simulated port isolation between the elements in the two-element array loaded only with GCLLs when they are excited in phase $\left(\left|\mathbf{S}_{13}\right|\right.$ and $\left.\left|\mathbf{S}_{24}\right|\right)$.

In contrast, when ports 2 and 4 are exited, the two-element behavior is switched into the orthogonal direction, which is now equivalent to the two patches being in an $H$-plane orientation [49]. As shown in Fig. 15(b), there are almost no evident currents distributed on the GCLLs. Referring to zones (2) and (3) in Fig. 6, the GCLLs are inserted into layer_2, which is below the radiating patch and, thus, the $E$-field in zone (2) dominates the coupling mechanism. As Fig. 6 then indicates, the electric field behavior will be orthogonal to the GCLLs and, as a result, they are not excited. Consequently, the simulated $\left|\mathrm{S}_{24}\right|$ values given in Fig. 16 remain almost the same as those shown in Fig. 14(b). Therefore, it is concluded that the GCLLs 
reduce the mutual coupling between $E$-plane oriented array elements since the $E$-fields they generate are oriented parallel to them.

\section{(2)Two-element array loaded only with $\pi$-shaped elements}

The surface current distributions on the traces of the two-element array loaded with only the three $\pi$-shaped elements are presented in Fig. 17. When ports 1 and 3 are excited with the same phase, elements 1 and 2 are in an $E$-plane orientation and create an $E$-field distribution similar to that in zone (1) of Fig. 6. Clearly, the $\pi$-shaped resonators cannot be excited in the operational bandwidth of those elements since under this condition the $E$-fields are orthogonal to them. As shown in Fig. 17(a), there is no apparent induced current on the $\pi$-shaped elements. Thus, as the $\left|S_{13}\right|$ values presented in Fig. 18 indicate, the port isolation results remain unchanged in comparison to the results given in Fig. 14(b) for the two-element array without the presence of the $\pi$-shaped elements.

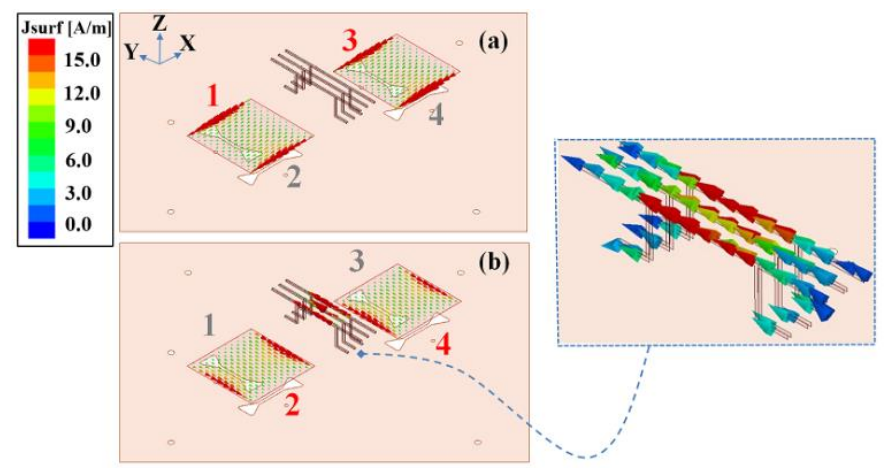

Fig. 17 Surface current distributions on the traces of the two-element array consisting of only elements No. 1 and No. 2 and loaded only with $\pi$-shaped elements. Excitation of (a) ports 1 and 3 only, and (b) ports 2 and 4 only.

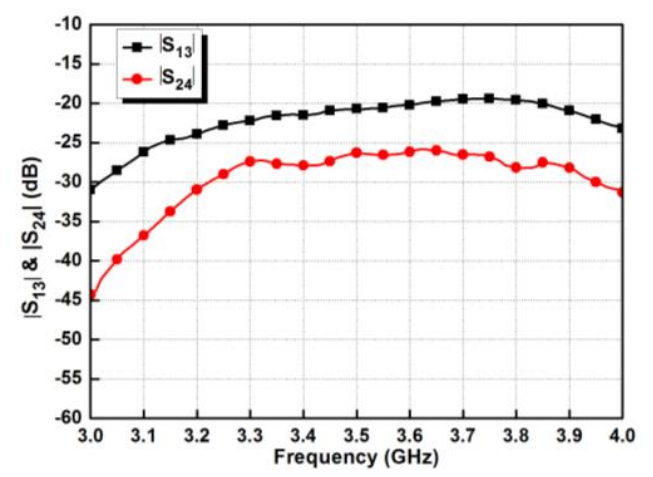

Fig. 18 Simulated port isolation between the elements in the two-element array loaded only with $\pi$-shaped elements when they are excited in phase $\left(\left|S_{13}\right|\right.$ and $\left.\left|\mathrm{S}_{24}\right|\right)$.

In contrast, when ports 2 and 4 are excited with the same phase, the two radiating elements are in an $H$-plane orientation and, consequently, exhibit a different coupling behavior. Referring again to Fig. 6, the radiating patch with $\sim \lambda_{0} / 2$ electric length along the $x$-axis creates the zone (2) (lower than the patch height) $E$-field behavior but with a half-period phase difference. In contrast, the $E$-field in the zone (3) (higher than the patch height) is polarized along the $y$-axis. This accommodates the excitation of the resonant $\pi$-shaped elements. Consequently, very strong induced currents are observed on the $\pi$-shaped elements. The mutual coupling levels indicated by the $\left|\mathrm{S}_{24}\right|$ values in Fig. 18 are decreased to $-25.83 \mathrm{~dB}$, witnessing a $7.47 \mathrm{~dB}$ reduction.

A further parametric study of the position of the $\pi$-shaped elements was performed. The height of the $\pi$-shaped elements $\left(h_{8}\right)$ was swept parametrically. These simulation results demonstrated that when $\mathrm{h}_{8}$ decreases from 15.5 to $8.0 \mathrm{~mm}$, which is equivalent to the transition from zone (3) to zone (2), the $\left|S_{24}\right|$ values increase significantly from -25.83 to $-20.96 \mathrm{~dB}$. This outcome further confirmed that the $\pi$-shaped elements operate well in zone (3), not in zone (2). Hence, it was concluded that the $\pi$-shaped elements have a significant impact on the reduction of the mutual coupling between the array elements along the $H$-plane, but they have little impact on the results when the array elements are oriented along the $E$-plane. In addition, by comparing the results in Figs. 13, 16 and 18, it is concluded that the presence of the $\pi$-shaped elements can significantly improve the decoupling performance of the GCLLs. The coupling level $\left|\mathrm{S}_{13}\right|$ witnesses a $\sim 17 \mathrm{~dB}$ reduction. In contrast, the GCLLs have little to no influence on the performance of the $\pi$-shaped elements, i.e., $\left|S_{24}\right|$ remains basically unchanged.

\section{EXPERIMENTAL VALIDATIONS}

A two-element array with both GCLLs and $\pi$-shaped elements was fabricated and tested. The experiments were performed to validate the basic design principles and the reported simulation results. We selected the two-element array consisting of elements No. 1 and No. 2 with the decoupling meta-structures reported in Fig. 9 as the proof-of-concept example.

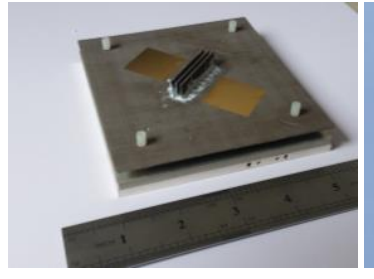

(a)

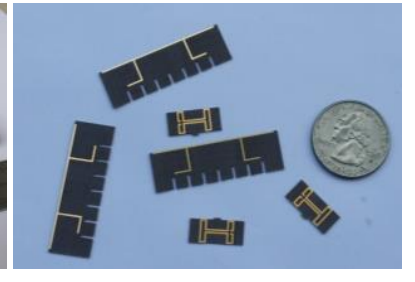

(b)
Fig. 19 The fabricated prototype of the two-element array consisting of elements No. 1 and No. 2 and loaded with both types of meta-structures. (a) 3-D view of the fabricated array, and (b) the fabricated GCLLs and $\pi$-shaped elements before installation.

The fabricated prototype after installation and the two types of decoupling structures before installation are depicted in Fig. 19. The array system was measured using an Agilent E8361A PNA vector network analyzer (VNA). The measured reflection coefficients and port isolation levels as functions of the source frequency are presented in Fig. 20. As shown in Fig. 20(a), the operational bandwidth, i.e., the frequencies for which the reflection coefficient $<-10 \mathrm{~dB}$, covers the entire range from 
3.3-3.6 GHz and, hence, the fractional bandwidth is $\sim 8.7 \%$. The mutual coupling levels between the cross polarization ports of each radiating element, i.e., $\left|S_{12}\right|$ for element No. 1 and $\left|S_{34}\right|$ for element No. 2, are lower than $-31.53 \mathrm{~dB}$. On the other hand, Fig. 20(b) depicts the isolation levels between the two elements. The mutual coupling levels for the same polarization ports $\left(\left|\mathrm{S}_{13}\right|\right.$ and $\left.\left|\mathrm{S}_{24}\right|\right)$ were suppressed to $-25.51 \mathrm{~dB}$, and those for the cross polarization ports $\left(\left|S_{14}\right|\right.$ and $\left.\left|S_{23}\right|\right)$ were increased slightly to $-34.33 \mathrm{~dB}$.

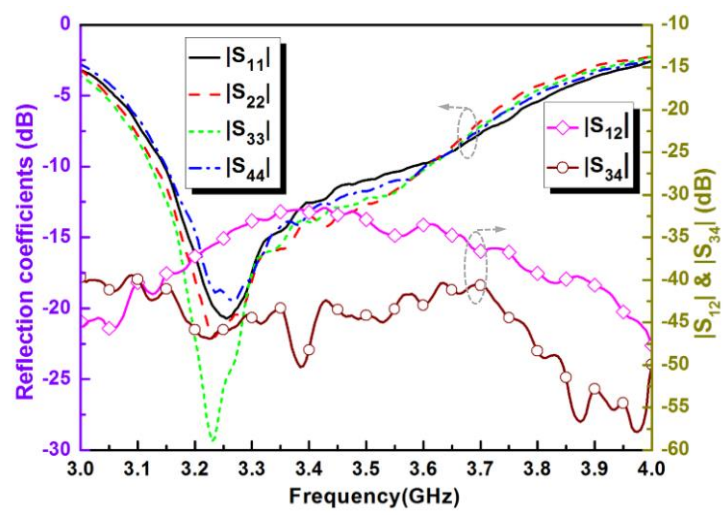

(a)

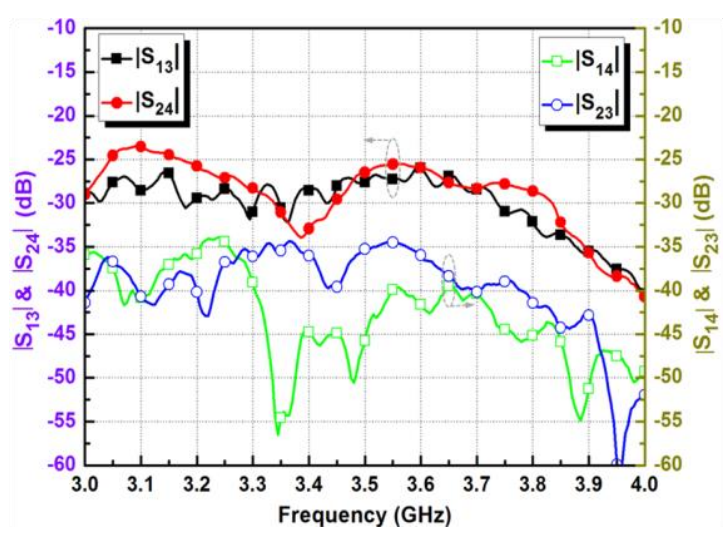

(b)

Fig. 20 Measured S-parameters of the two-element array consisting of elements No. 1 and No. 2 and loaded with both types of meta-structures. (a) Values of the reflection coefficients and isolation levels between the ports for each antenna element, and (b) isolation levels between the two antenna elements.

On the whole, good agreement is obtained between the measured and simulated results. The small differences, of course, arose from the unavoidable errors in the fabrication, assembly, and measurement. They were deemed to be very acceptable. It also should be noted that in order to make the CLLs well-connected to the ground plane in the experiment, epoxy resins and hardeners were utilized in the installation process. These were not included in the simulation and also account for some of the small differences.

The far-field radiation performance characteristics of the two-element array were also investigated numerically and experimentally. The experiments were carried out in an anechoic chamber at the University of Electronic Science and Technology of China (UESTC). The chamber consists of an AgilentN5230A PNA-L VNA and a SATIMO passive measurement system. The measured results and the relevant simulation values at $3.45 \mathrm{GHz}$, when only port 1 or port 2 is excited, are presented in Fig. 21.

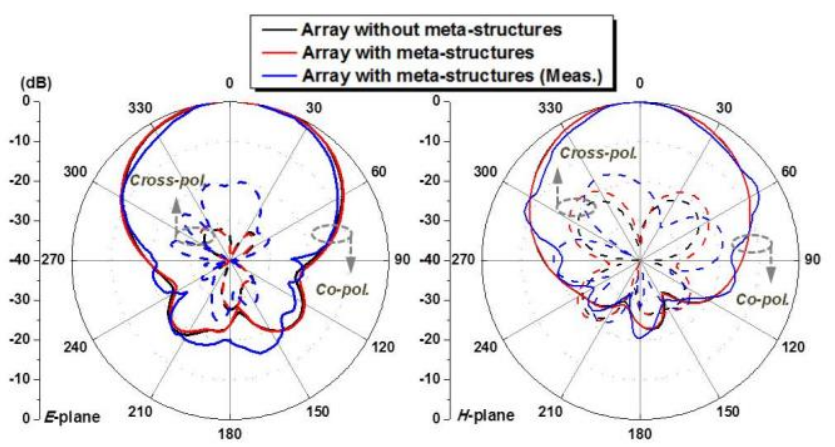

(a)

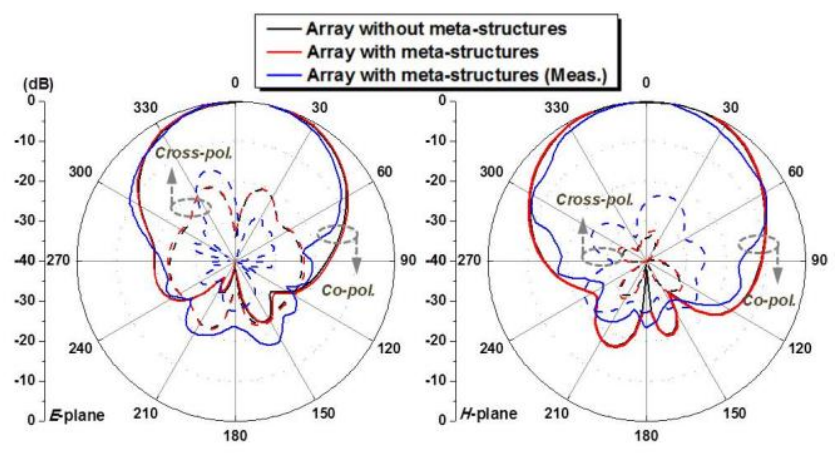

(b)

Fig. 21 Simulated and measured radiation patterns of the two-element array consisting of elements No. 1 and No. 2 with and without the meta-structures at $3.45 \mathrm{GHz}$, (a) in the $E-(\mathrm{ZOX}-)$ and $H$ - (ZOY-) planes when only port 1 is excited, and (b) in the $E$ - (ZOY-) and $H$ - (ZOX-) planes when only port 2 is excited.

As is illustrated in Fig. 21 (a), when only port 1 is excited at the resonance frequency: $3.45 \mathrm{GHz}$, the measured (simulated) peak realized gain reaches $8.73 \mathrm{dBi}(8.31 \mathrm{dBi})$, and the front-to-back ratio (FTBR) value is $20.16 \mathrm{~dB}(23.37 \mathrm{~dB})$. The corresponding half-beam coverage is from $-27^{\circ}$ to $40^{\circ}$ ( $-38^{\circ}$ to $\left.45^{\circ}\right)$ in the $E$-plane and from $-29^{\circ}$ to $23^{\circ}\left(-37^{\circ}\right.$ to $\left.32^{\circ}\right)$ in the $H$-plane. On the other hand, when only port 2 is excited at its resonance frequency: $3.45 \mathrm{GHz}$, the measured (simulated) peak realized gain value is $8.02 \mathrm{dBi}(7.64 \mathrm{dBi})$, and the FTBR value is 23.49 (41.72) $\mathrm{dB}$. The measured (simulated) half-beam coverage in the $E$ - and $H$-planes is, respectively, in the interval from $-27.5^{\circ}$ to $38^{\circ}$ (from $-32^{\circ}$ to $43^{\circ}$ ) and from $-35^{\circ}$ to $33^{\circ}$ (from $-46^{\circ}$ to $46^{\circ}$ ). Additionally, the measured (simulated) overall radiation efficiencies and polarization purity in both of these cases were as high as $84.6 \%$ and $16 \mathrm{~dB}(92.5 \%$ and 20 $\mathrm{dB})$. Again, the small differences between the measured and simulated values are ascribed to the usual unavoidable errors introduced in the fabrication, assembly, and measurement processes. Note that by comparing the simulated radiation patterns of the array with and without the meta-structures, it is demonstrated further that while the presence of the meta-structures significantly improved the mutual coupling levels, they had little effect on the peak gain values, radiation efficiencies, half-beamwidths, cross-polarization levels, and FTBRs. 


\section{CONCLUSIONS}

The presence of the combination of two types of resonant meta-structures, i.e., the GCLLs and $\pi$-shaped elements, has been demonstrated as an effective mutual coupling reduction strategy for dual-polarized patch antenna arrays. The operational mechanisms of both resonators were investigated in detail. By integrating the meta-structures into a high-density, triangular lattice patch array, the mutual coupling between adjacent elements oriented in both the $E$ - and $H$-planes can be suppressed significantly. A proof-of-concept prototype was fabricated and experiments were performed to confirm the design principles. The simulated and measured results were in very good agreement. They validate the effectiveness of the optimally designed meta-structures in mutual coupling suppression within two-dimensional triangular lattice patch antenna arrays. Benefitting from their increased data capacity, decreased spatial correlations, and reduced SINRs, this class of arrays is expected to have a very important role in several anticipated 5G MIMO applications. The $-25 \mathrm{~dB}$ and better isolation realized with the presence of the meta-structures is considered to be a level necessary to achieve those applications. Nevertheless, because of the overall 3-D nature of the composite meta-structure, more effort is currently being expended to achieve a simpler planar version for easy integration with a wider variety of antenna arrays for more widespread engineering applications in the near future.

\section{APPENDIX}

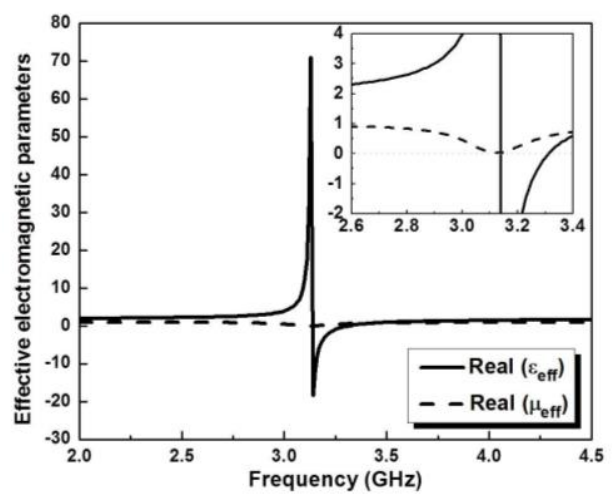

Fig. 22 The retrieved effective medium parameters of the GCLL unit cell shown in Fig. 1.

In order to further explain the dominant electric responses of the two types of meta-structures, their effective medium parameters were retrieved from the simulated S-parameters, whose magnitudes are given in Figs. 2(a) and 4(a), respectively [50]. The corresponding results for the unit cells of the resonant GCLL and the $\pi$-shaped elements are presented in Figs. 22 and 23 , respectively. It is clear that, in general, the real part of the effective permittivity is negative and the real part of the effective permeability is positive above $3.1 \mathrm{GHz}$. These results indicate that both types of resonators are dominated by their electric responses and that they exhibit stop-bands (because the corresponding real part of their index of refraction is imaginary) for the frequencies of interest. Note that Fig. 23 indicates the effective permeability is negative and the permittivity is positive near $2.8 \mathrm{GHz}$. This phenomenon is ascribed to the bi-anisotropically induced magnetic response behavior from the $\pi$-shaped elements themselves [51].

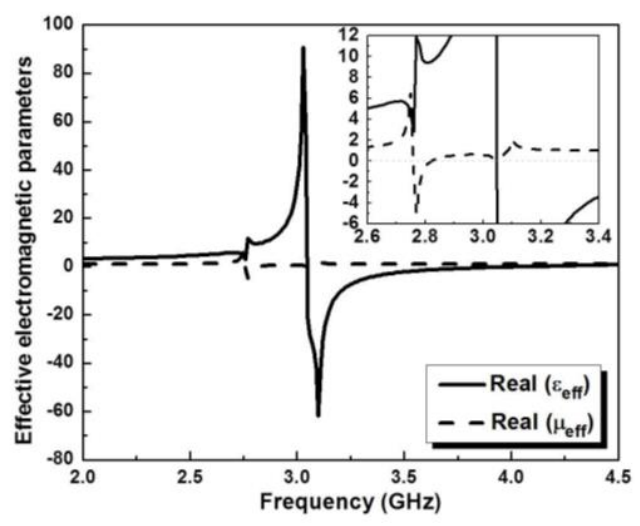

Fig. 23 The retrieved effective medium parameters of the unit cell of the $\pi$-shaped element shown in Fig. 3.

\section{References}

[1] L. Dong, H. Choo, R. W. H. Jr., and H. Ling, "Simulation of MIMO channel capacity with antenna polarization diversity," IEEE Trans. Wireless Commun., vol. 4, no. 4, pp. 1869-1873, Apr. 2005.

[2] S. Biswas, C. Masouros, and T. Ratnarajah, "Performance analysis of large multiuser MIMO systems with space-constrained 2-D antenna arrays," IEEE Trans. Wireless Commun., vol. 15, no. 5, pp. 3492-3505, May 2016.

[3] S. Soltani, and R. D. Murch, "A compact planar printed MIMO antenna design," IEEE Trans. Antennas Propag., vol. 63, no. 3, pp. 1140-1149, Mar. 2015.

[4] C. A. Balanis, Antenna Theory: Analysis and Design, 3rd edition, New York: Wiley Interscience, 2005.

[5] Z. Li, Z. Du, M. Takahashi, K. Saito, and K. Ito, "Reducing mutual coupling of MIMO antennas with parasitic elements for mobile terminals," IEEE Trans. Antennas Propag., vol. 60, no. 2, pp. 473-481, Feb. 2012.

[6] B. K. Lau, and J. B. Andersen, "Simple and efficient decoupling of compact arrays with parasitic scatterers," IEEE Trans. Antennas Propag., vol. 60, no. 2, pp. 464-472, Feb. 2012.

[7] M.-C. Tang, S. Xiao, T. Deng, and B.-Z. Wang, "Parasitic patch of the same dimensions enabled excellent performance of microstrip antenna array," Appl. Comput. Electromagn. Soc. J., vol. 25, No. 10, pp. 862 866, 2010.

[8] F. Yang, and Y. Rahmat-Samii, "Microstrip antennas integrated with electromagnetic band-gap (EBG) structures: A low mutual coupling design for array applications," IEEE Trans. Antennas Propag., vol. 51, no. 10, pp. 2936-2946, Oct. 2003

[9] G. Expósito-Domínguez, J.-M. Fernández-Gonzalez, P. Padilla, and M. Sierra-Castañer, "Mutual coupling reduction using EBG in steering antennas," IEEE Antennas Wirel. Propag. Lett., vol. 11, pp.1265-1268, 2012.

[10] H. S. Farahani, M. Veysi, M. Kamyab, and A. Tadjalli, "Mutual coupling reduction in patch antenna arrays using a UC-EBG superstrate," IEEE Antennas Wirel. Propag. Lett., vol. 9, pp. 57-59, 2010.

[11] M. J. Al-Hasan, T. A. Denidni, and A. R. Sebak, "Millimeter-wave compact EBG structure for mutual coupling reduction applications," IEEE Trans. Antennas Propag., vol. 63, no. 2, pp. 823-828, Feb. 2015.

[12] C.-Y. Chiu, C.-H. Cheng, R. D. Murch, and C. R. Rowell, "Reduction of mutual coupling between closely-packed antenna elements," IEEE Trans. Antennas Propag., vol.55, no. 6, pp. 1732-1738, Jun. 2007.

[13] S. Xiao, M.-C. Tang, Y.-Y. Bai, S. Gao, B.-Z. Wang, "Mutual coupling suppression in microstrip array using defected ground structure," IET 
Microw. Antennas Propag., vol. 5, no. 12, pp. 1488 - 1494, Sep. 2011.

[14] S. Zhang, B. K. Lau, Y. Tan, Z. Ying, and S. He, "Mutual coupling reduction of two PIFAs with a T-shape slot impedance transformer for MIMO mobile terminals," IEEE Trans. Antennas Propag., vol. 60, no. 3, pp. 1521-1531, Mar. 2012.

[15] L. Qu, R. Zhang, and H. Kim, "Decoupling between ground radiation antennas with ground-coupled loop-type isolator for WLAN applications," IET Microw. Antennas Propag., vol. 10, iss. 5, pp. 546 $552,2016$.

[16] M.-C. Tang, S. Xiao, T. Deng, and B.-Z. Wang, "Novel folded single split ring resonator and its application to eliminate scan blindness in infinite phased array," 2010 International Symposium on Signals, Systems and Electronics (ISSSE2010), pp. 1-4, Nanjing China, Sep. 17 - 20, 2010.

[17] M.-C. Tang, S. Xiao, B.-Z. Wang, J. Guan, and T. Deng, "Improved performance of a microstrip phased array using broadband and ultra- lowloss metamaterial slabs," IEEE Antennas Propag. Mag., vol. 53, no. 6, pp. 31 - 41, Dec. 2011.

[18] D. B. M. Trindade, C. Müller, M. C. F. D. Castro, and F. C. C. D. Castro, "Metamaterials applied to ESPAR antenna for mutual coupling reduction," IEEE Antennas Wirel. Propag. Lett., vol. 14, pp. 430-433, 2015.

[19] Z. Qamar, U. Naeem, S. A. Khan, M. Chongcheawchamnan, and M. F. Shafique, "Mutual coupling reduction for high-performance densely packed patch antenna arrays on finite substrate," IEEE Trans. Antennas Propag., vol. 64, no. 5, pp. 1653-1660, May 2016.

[20] R. Hafezifard, M. Naser-Moghadasi, J. R. Mohassel, and R. A. Sadeghzadeh, "Mutual coupling reduction for two closely spaced meander line antennas using metamaterial substrate," IEEE Antennas Wirel. Propag. Lett., vol. 15, pp. 40-43, 2016.

[21] K. Buell, H. Mosallaei, and K. Sarabandi, "Metamaterial insulator enabled superdirective array," IEEE Trans. Antennas Propag., vol. 55, no. 4, pp. 1074-1085, Apr. 2007.

[22] B. Wu, H. Chen, J. A. Kong, and T. M. Grzegorczyk, "Surface wave suppression in antenna systems using magnetic metamaterial," J. Appl. Phys., vol.101, pp. 1129 13(1-4), Jun. 2007.

[23] X. M. Yang, X. G. Liu, X. Y. Zhou, and T. J. Cui, "Reduction of mutual coupling between closely packed patch antennas using waveguided metamaterials," IEEE Antennas Wirel. Propag. Lett., vol. 11, pp. 389-391, 2012.

[24] M.-C. Tang, S.-Q. Xiao, J. Guan, Y.-Y. Bai, S.-S. Gao, and B.-Z. Wang, "Composite metamaterial enabled excellent performance of microstrip antenna array," Chin. Phys. B, vol. 19, no. 7, 074214, Jul., 2010.

[25] J.-Y. Lee, S.-H. Kim, and J.-H. Jang, "Reduction of mutual coupling in planar multiple antenna by using 1-D EBG and SRR structures," IEEE Trans. Antennas Propag., vol. 63, no. 9, pp. 4194-4198, Sep. 2015.

[26] P. J. Ferrer, J. M. González-Arbesú, and J. Romeu, "Decorrelation of two closely spaced antennas with a metamaterial AMC surface," Microw. Opt. Technol. Lett., vol. 50, no. 5, pp. 1414-1417, May 2008.

[27] M. Imbert, P. J. Ferrer, J. M. González-Arbesú, and J. Romeu, "Assessment of the performance of a metamaterial spacer in a closely spaced multiple-antenna system," IEEE Antennas Wirel. Propag. Lett., vol. 11, pp. 720-723, 2012.

[28] G. Zhai, Z. N. Chen, and X. Qing, "Enhanced isolation of a closely spaced four-element MIMO antenna system using metamaterial mushroom," IEEE Trans. Antennas Propag., vol. 63, no. 8, pp. 3362-3370, Aug. 2015.

[29] H. Qi, L. Liu, X. Yin, H. Zhao, and W. J. Kulesza, "Mutual coupling suppression between two closely spaced microstrip antennas with an asymmetrical coplanar strip wall," IEEE Antennas Wirel. Propag. Lett., vol. 15, pp. 191-194, 2016.

[30] S.-W. Su, C.-T. Lee, and F.-S. Chang, "Printed MIMO-Antenna system using neutralization-line technique for wireless USB-dongle applications," IEEE Trans. Antennas Propag., vol. 60, no. 2, pp. 456-463, Feb. 2012.

[31] Y. Yu, L. Yi, X. Liu, and Z. Gu, "Compact dual-frequency microstrip antenna array with increased isolation using neutralization lines," Progress In Electromagnetics Research Letters, vol. 56, pp. 95-100, 2015.

[32] S. Zhang and G. F. Pedersen, "Mutual coupling reduction for UWB MIMO antennas with a wideband neutralization line," IEEE Antennas Wirel. Propag. Lett., vol. 15, pp. 166-169, 2016.

[33] S. M. Amjadi, and K. Sarabandi, "Mutual coupling mitigation in broadband multiple-antenna communication systems using feedforward technique," IEEE Trans. Antennas Propag., vol. 64, no. 5, pp. 1642-1652, May 2016.
[34] J. C. Coetzee and Y. Yu, "New modal feed network for a compact monopole array with isolated ports", IEEE Trans. Antennas Propag., vol. 56, no.12, pp.3872-3875, Dec. 2008.

[35] K.-C. Lin, C.-H. Wu, C.-H. Lai, and T.-G. Ma, "Novel dual-band decoupling network for two-element closely spaced array using synthesized microstrip lines," IEEE Trans. Antennas Propag., vol. 60, no.11, pp.5118-5128, Nov. 2012.

[36] L. Zhao, L. K. Yeung, and K. -L. Wu, "A coupled resonator decoupling network for two-element compact antenna arrays in mobile terminals," IEEE Trans. Antennas Propag., vol. 62, no.5, pp.2767-2776, May 2014.

[37] R.-L. Xia, S.-W. Qu, P.-F. Li, D.-Q. Yang, S. Yang, and Z.-P. Nie, "Wide-angle scanning phased array using an efficient decoupling network," IEEE Trans. Antennas Propag., vol. 63, no. 11, pp. 5161-5165, Nov. 2015.

[38] C.-H. Wu, C.-L. Chiu, and T.-G. Ma, "Very compact fully lumped decoupling network for a coupled two-element array," IEEE Antennas Wirel. Propag. Lett., vol. 15, pp. 158-161, 2016.

[39] J.-Y. Pang, S.-Q. Xiao, Z.-F. Ding, and B.-Z. Wang, "Two-element PIFA antenna system with inherent performance of low mutual coupling," IEEE Antennas Wirel. Propag. Lett., vol. 8, pp. 1223-1226, 2009.

[40] K. L. Chung, and S. Kharkovsky, "Mutual coupling reduction and gain enhancement using angular offset elements in circularly polarized patch array ," IEEE Antennas Wirel. Propag. Lett., vol. 12, pp. 1122-1124, 2013.

[41] J. Li, S. Yang, Y. Gou, J. Hu, and Z. Nie, "Wideband dual-polarized magnetically coupled patch antenna array with high port isolation," IEEE Trans. Antennas Propag., vol. 64, no.1, pp.3872-3875, Jan. 2016.

[42] ANSYS/ANSOFT High Frequency Structure Simulation (HFSS), ver. 15.0, ANSYS Corp. [Online]. Available at www.ansoft.com.

[43] D. R. Smith, Willie J. Padilla, D. C. Vier, S. C. Nemat-Nasser, and S. Schultz, "Composite medium with simultaneously negative permeability and permittivity" Phys. Rev. Lett., vol. 84, no.18, pp. 4184-4187, May 2000.

[44] N. Engheta and R. W. Ziolkowski, Metamaterials: Physics and Engineering Explorations, Piscataway, N.J.: IEEE-Wiley Press, 2006.

[45] P. Gay-Balmaz and O. J. F. Martin, "Electromagnetic resonances in individual and coupled split-ring resonators," J. Appl. Phys., vol. 92, no. 5, pp. 2929-2936, Sep. 2002.

[46] S. D. Targonski and D. M. Pozar, "Design of wideband circularly polarized aperture coupled microstrip antennas," IEEE Trans. Antennas Propag., vol. 41, no. 2, pp. 214-220, Feb.1993.

[47] W. Zhu, S. Xiao, R. Yuan, and M. -C. Tang, "Broadband and dual circularly polarized patch antenna with $\mathrm{H}$-shaped aperture," Proceedings of 2014 International Symposium on Antennas and Propagation (ISAP2014), pp. 549-550, Kaohsiung, Taiwan, Dec. 2-5, 2014.

[48] I. J. Bhal and P. Bhartia, Microstrip Antenna. Dedham, MA: Artech House, 1980.

[49] A. H. Mohammadian, N. M. Martin, and D. W. Griffin, "A theoretical and experimental study of mutual coupling in microstrip antenna arrays," IEEE Trans. Antennas Propag., vol. 37, no. 10, pp. 1217-1223, Oct. 1989.

[50] D. R. Smith, D. C. Vier, Th. Koschny, C. M. Soukoulis, "Electromagnetic parameter retrieval from inhomogeneous metamaterials," Phys. Rev. E, vol. 71, pp.036617 (1-11), Mar. 2005.

[51] J. D. Baena, J. Bonache, F. Martín, R. M. Sillero, F. Falcone, T. Lopetegi, M. A. G. Laso, J. G.-G., I. Gil, M. F. Portillo, and M. Sorolla, "Equivalent-circuit models for split-ring resonators and complementary split-ring resonators coupled to planar transmission lines," IEEE Trans. Microw. Theory Tech., vol. 53, no. 4, pp.1451-1460, Apr. 2005.

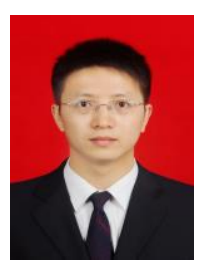

Ming-Chun Tang (S'12-M'13-SM'16) received the B. S. degree in physics from the Neijiang Normal University, Neijiang, China, in 2005 and the Ph. D. degree in radio physics from the University of Electronic Science and Technology of China (UESTC), in 2013. From August 2011 to August 2012, he was also with the Department of Electrical and Computer Engineering, The University of Arizona, Tucson, AZ, USA, as a Visiting Scholar. He is currently an Assistant Professor in the College of Communication Engineering, Chongqing University, China. His research interests include electrically small antennas, RF circuits, metamaterial designs and their applications.

Prof. Tang was a recipient of the Best Student Paper Award in the 2010 
International Symposium on Signals, Systems and Electronics (ISSSE2010) held in Nanjing, China. He is the founding Chair of the IEEE AP-S / MTT-S Joint Chongqing Chapter. He serves on the Editorial Boards of several journals, including International Journal of Antennas and Propagation, Journal of Electrical Engineering, and MAYFEB Journal of Electrical and Electronic Engineering. He has also served on the review boards of many journals, including the IEEE Transactions on Antennas and Propagation, IEEE Transactions on Microwave Theory and Techniques, IEEE Antennas and Wireless Propagation Letters, IEEE Antennas and Propagation Magazine, IEEE Microwave and Wireless Components Letters, IEEE Access, and many international conferences as a General Chair, TPC Member, Session Organizer, and the Session Chair.

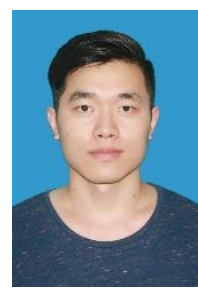

Zhiyuan Chen (S'16) received the B.S. degree in electronic science and technology from the Shandong Normal University (SDNU), Jinan, China, in 2015. He is currently pursuing the M.S. degree in electronics and communication engineering at Chongqing University, Chongqing, China.

His research interests include ultra-wideband antennas, planar antennas and arrays.

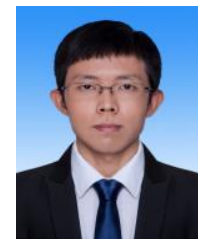

Hao Wang (S'16) received the B.S. degree in communication engineering from the Lanzhou University (LZU), Lanzhou, China, in 2014.He is currently pursuing the M.S. degree in electronic science and technology at Chongqing University, Chongqing, China.

His research interests include electrically small antennas, planar antennas and arrays.

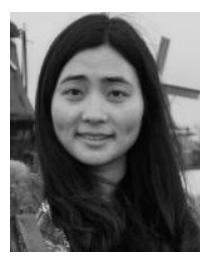

Mei Li (M'16) received the Ph.D. in radio physics from the University of Electronic Science and Technology of China, Chengdu, in 2016. From 2014 to 2016, she was with the Applied Electromagnetics Research Group, University of California at San Diego, San Diego, CA, USA, as a Visiting Graduate. She is currently with Chongqing University. Her current research interests include metasurfaces, antennas and arrays.

Bing Luo, photograph and biography not available at the time of publication.

Jiadi Wang, photograph and biography not available at the time of publication.

Zhongli Shi, photograph and biography not available at the time of publication.

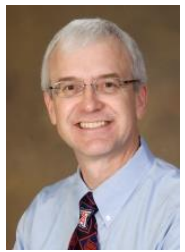

Richard W. Ziolkowski (M'87-SM'91-F'94) received the B.Sc. (magna cum laude) degree (Hons.) in physics from Brown University, Providence, RI, USA, in 1974; the M.S. and Ph.D. degrees in physics from the University of Illinois at Urbana-Champaign, Urbana, IL, USA, in 1975 and 1980, respectively; and the Honorary Doctorate degree from the Technical University of Denmark, Kongens Lyngby, Denmark in 2012.

He is currently a Distinguished Professor with the University of Technology Sydney, Global Big Data Technologies Centre, Ultimo NSW, Australia. He is also a Litton Industries John M. Leonis Distinguished Professor with the Department of Electrical and Computer Engineering and a Professor with the College of Optical Sciences at The University of Arizona. He was the
Computational Electronics and Electromagnetics Thrust Area Leader with the Lawrence Livermore National Laboratory, Engineering Research Division, before joining The University of Arizona, Tucson, AZ, USA, in 1990. He was the Australian DSTO Fulbright Distinguished Chair in Advanced Science and Technology from 2014-2015. He was a 2014 Thomas-Reuters Highly Cited Researcher. His current research interests include the application of new mathematical and numerical methods to linear and nonlinear problems dealing with the interaction of electromagnetic and acoustic waves with complex linear and nonlinear media, as well as metamaterials, metamaterial-inspired structures, and applications-specific configurations.

Prof. Ziolkowski is a Fellow of the Optical Society of America (OSA, 2006), and of the American Physical Society (APS, 2016). He served as the President of the IEEE Antennas and Propagation Society in 2005. He is also actively involved with the URSI, OSA and SPIE professional societies. 